\title{
Secure Multiparty Computation with General Interaction Patterns
}

\author{
Shai Halevi \\ IBM Research, USA
}

\author{
Yuval Ishai \\ Technion, Israel and UCLA
}

\author{
Abhishek Jain \\ Johns Hopkins University
}

\author{
Eyal Kushilevitz \\ Technion, Israel
}

\author{
Tal Rabin \\ IBM Research, USA
}

\begin{abstract}
We present a unified framework for studying secure multiparty computation (MPC) with arbitrarily restricted interaction patterns such as a chain, a star, a directed tree, or a directed graph. Our study generalizes both standard MPC and recent models for MPC with specific restricted interaction patterns, such as those studied by Halevi et al. (Crypto 2011), Goldwasser et al. (Eurocrypt 2014), and Beimel et al. (Crypto 2014).

Since restricted interaction patterns cannot always yield full security for MPC, we start by formalizing the notion of "best possible security" for any interaction pattern. We then obtain the following main results:
\end{abstract}

- Completeness theorem. We prove that the star interaction pattern is complete for the problem of MPC with general interaction patterns.

- Positive results. We present both information-theoretic and computationally secure protocols for computing arbitrary functions with general interaction patterns. We also present more efficient protocols for computing symmetric functions, both in the computational and in the information-theoretic setting.

Our computationally secure protocols for general functions necessarily rely on indistinguishability obfuscation while the ones for computing symmetric functions make simple use of multilinear maps.

- Negative results. We show that, in many cases, the complexity of our information-theoretic protocols is essentially the best that can be achieved.

All of our protocols rely on a correlated randomness setup, which is necessary in our setting (for computing general functions). In the computational case, we also present a generic procedure to make any correlated randomness setup reusable, in the common random string model.

Although most of our information-theoretic protocols have exponential complexity, they may be practical for functions

Permission to make digital or hard copies of all or part of this work for personal or classroom use is granted without fee provided that copies are not made or distributed for profit or commercial advantage and that copies bear this notice and the full citation on the first page. Copyrights for components of this work owned by others than ACM must be honored. Abstracting with credit is permitted. To copy otherwise, or republish, to post on servers or to redistribute to lists, requires prior specific permission and/or a fee. Request permissions from Permissions@ acm.org.

ITCS'16, January 14-16, 2016, Cambridge, MA, USA

(C) 2016 ACM. ISBN 978-1-4503-4057-1/16/01 ...\$15.00.

DOI: http://dx.doi.org/10.1145/2840728.2840760. on small domains (e.g., $\{0,1\}^{20}$ ), where they are concretely faster than their computational counterparts.

\section{INTRODUCTION}

Secure multiparty computation (MPC) allows $n$ mutually suspicious parties to evaluate a function on their joint inputs in such a manner that no information about their inputs, beyond the output of the computation, is revealed to each other. Since the first general feasibility results for MPC 43 . 28, 5, 12, almost all prior work in this area has considered protocols that require full interaction between the parties. Such protocols typically proceed in rounds, where in each round each party may send messages to all other parties, thus requiring that all parties remain online throughout the execution of the protocol.

MPC with Restricted Interaction. Full interaction between the parties is often problematic, and in many scenarios, simply infeasible. For instance, physical distances between wireless devices may prevent them from directly communicating with each other, and temporal constraints may restrict their availability to send or receive messages (e.g. due to battery life). Furthermore, efficiency considerations may also motivate a leaner form of communication. Consider, for instance, the goal of computing the majority vote over the inputs of $n$ parties. While this task can be performed using only $(n-1)$ messages if no security is needed, typical MPC protocols with full interaction involve $\Omega\left(n^{2}\right)$ point-to-point messages to compute the same task securely.

Such considerations have motivated the study of MPC protocols with restricted interaction between the parties. Halevi, Lindell, and Pinkas 33. were the first to study this problem: they consider an interaction pattern where each of the $n$ parties, in an ordered fashion, sends a single message to a central server, who eventually computes the output of the function. A different interaction pattern, where each party independently (i.e., without any predetermined order) sends a single message to the server, was recently considered by Goldwasser et al. 29 and Beimel et al. 3. In both cases, the security guarantee is necessarily weaker than the standard simulation-based security for MPC (see below for further discussion on security).

The above works constitute two specific examples of restricted interaction patterns. In general, different application scenarios may dictate different interaction patterns. For example, applications involving data aggregation typically use interaction patterns that can be represented as a directed tree. Furthermore, the topology of the communication network (used by an application) may itself limit the 
choices of interaction patterns: e.g., a communication network without any node with full degree is not consistent with server-centered interaction patterns. Importantly, as we discuss below, the security guarantees that we get would typically depend on the interaction pattern at hand.

Our Goal: MPC with General Interaction Patterns. Seeking to understand the fundamental role of interaction patterns in MPC, we study the problem of MPC with arbitrarily restricted or general interaction patterns. We ask the following broad question:

Given an arbitrary $n$-party interaction pattern $\mathcal{I}$ and an $n$-input function $f$, can $f$ be securely realized by a protocol that complies with $\mathcal{I}$ ? If so, how efficiently and under what assumptions?

Before addressing this question, we should clarify how we model interaction patterns, what we mean by "securely," and what setup assumptions we are willing to make.

Modeling Interaction Patterns. A natural starting approach is to represent an interaction pattern as a directed acyclic graph (DAG), where each node represents a party who expects to receive messages from all of its parents and can then send messages to all of its children, and where the sinks of the graph compute outputs. Two simple examples of DAGs include a chain, a simple directed path traversing all nodes, and a star, a graph connecting all nodes to a single central node. The protocols from 33,32 can be adapted to accommodate a chain-based interaction, whereas the protocols from 29, 3] were designed for the case of a star-based interaction. More general DAG-based interaction patterns naturally arise in "self-forming sensor networks," where multiple sensor nodes form an arbitrary communication graph and then collect and compute on data that is transmitted, using the smallest possible number of messages, to a central base station.

While DAGs are an important special case, general interaction patterns are not necessarily restricted to DAGs. Some other useful patterns include the server-centered interaction pattern from [33, a two-way chain, where messages travel along a chain from $P_{n}$ to $P_{1}$ and back to $P_{n}$ who computes an output, or the traditional multi-round protocols over a fully-connected point-to-point network. In the full paper we describe a unified modeling of general interaction patterns.

\subsection{Formulating Achievable Security}

In the traditional model of MPC, the corrupted parties are restricted to learning the outputs of $f$ on just a single input $\left(x_{1}, \ldots, x_{n}\right)$. However, as observed by [33], this property cannot always be achieved in the case of restricted interaction patterns. For instance, in the server-centered interaction pattern of [33], if both the server and the last few parties $P_{i}, \ldots, P_{n}$ are corrupted, there is nothing to prevent the adversary from learning the value of $f$ on the honest inputs $x_{1}, \ldots, x_{i-1}$ and every possible choice of corrupted inputs $x_{i}^{*}, \ldots, x_{n}^{*}$.

To define the "best possible security" for a fixed interaction pattern, we use the notions of free and fixed inputs and residual function from 33. We call the inputs that the adversary can vary the free inputs, and the other inputs are the fixed inputs. Clearly, all the honest parties' inputs are fixed. Crucially, however, some of the inputs controlled by the adversary can be fixed as well. For example, in the protocols from 33], the only free inputs are those of corrupted parties that send messages after the last honest party does; the inputs of all other corrupted parties are fixed.

Extending the model from 33 to our setting of general interaction patterns, we first consider the case where only one party computes an output and call this party the evaluator. The input of a corrupted party $P_{i}$ is considered fixed if the interaction pattern includes any message path that leads from $P_{i}$ to the evaluator and passes through some honest party. The input of a corrupted $P_{i}$ is free if all paths from $P_{i}$ to the evaluator consist only of other corrupted parties. For example, in a star pattern with a corrupted center, the inputs of all the corrupted parties are free 29, 3. In contrast, traditional MPC requires the inputs of all corrupted parties to be fixed.

The "best possible security" is defined by the residual function, that captures everything that the adversary can learn about the honest parties' by restricting $f$ to the values of all the fixed inputs while allowing arbitrary choices of the free inputs. Some additional subtleties arise in the maliciousadversary model when multiple parties compute outputs; in particular, security in this case generalizes goals such as Byzantine agreement. We discuss these issues further in the full paper leaving the issue of malicious-security with multiple output nodes to future work.

An important technical point relates to the achievable notion of simulation. Traditional ideal-vs-real definitions of security (cf. 31, 9, 27]) require efficient simulation, but it is known that efficient simulation is, in general, impossible in our setting 33, 29]. For example, protocols with efficient simulation for a star (or even chain) interaction pattern imply virtual black-box obfuscation 29, which is known to be impossible in general [2]. To get around these impossibility results, we settle for security with respect to indistinguishability or unbounded simulation.

Correlated randomness setup. It is not hard to see that without any form of setup, even very simple functions such as majority cannot always be realized with any meaningful notion of security. Consider for instance the star pattern: here, all the adversary's inputs are free; as such, the adversary can simply learn the residual function in the information-theoretic setting in the absence of any setup 3. 1

Perhaps the simplest model to circumvent such impossibility results is the "minimal model" (PSM) from 21] with general correlated randomness setup, where the parties have access to a source $\left(r_{1}, \ldots, r_{n}\right)$ of correlated random strings. When implementing such protocols, the correlated randomness can come from a trusted dealer or generated using an offline MPC protocol that takes place before the inputs are known and before the limitations on interaction are imposed. This clean model is popular both for a theoretical study of MPC and as a platform for practical implementations that exploit the efficiency benefits of offline preprocessing. (See 6, 16, 3, 17, and references therein.)

The correlated randomness setup can either be reusable or non-reusable. Namely, it can either be akin to a onetime pad, allowing only a single run of the protocol (which is typically the case for protocols with information-theoretic security), or it can allow polynomial number of runs (which is sometimes possible in the computational setting). A key advantage of the correlated randomness setup model is that

\footnotetext{
${ }^{1}$ In the computational setting, such an attack can be applied to learnable functions.
} 
we can hope to tolerate any number of corrupted parties even in the information-theoretic setting 37, 35, 6.

Is "Best Possible Security" Good Enough? Though weaker than the standard notion of security for MPC protocols, our notion of "best possible security" is still meaningful in many interesting cases. First, depending on the interaction pattern and the set of corrupted parties, it could be that most corrupted parties are fixed and hence the residual function is quite degenerate (or even all inputs are fixed as in standard MPC). Second, there are functions for which access even to a "large" residual function does not compromise the secrecy of uncorrupted inputs significantly. Examples include symmetric functions (such as majority) where the size of the residual truth table is not very significant (see 3 for a discussion), as well as unlearnable functions where it is computationally hard to figure out the inputs of honest parties even when given a polynomially large residual function.

\subsection{Our Results}

We give a variety of answers to the main question posed above. Our results come in three different flavors: (a) "lowend" protocols that offer unconditional security and are generally exponential in the input size (except for special function classes and special interaction patterns); (b) "high-end" protocols that (necessarily) use general-purpose obfuscation techniques to achieve polynomial-time solutions for general functions and interaction patterns; and (c) "mid-range" protocols that make simple use of multilinear maps to compute symmetric functions with general interaction patterns.

Our protocols tolerate an arbitrary number of corrupted parties in the static corruption model. Below, when describing our results, we use the phrase $\mathcal{I}$-compliant to denote that the interaction pattern in a protocol is consistent with $\mathcal{I}$.

I. A Completeness Theorem for Interaction Patterns. In Section 2 we show that the star interaction pattern is complete for secure computation with restricted interaction patterns. Specifically, we give an efficient, unconditional reduction from the problem of realizing a function $f$ using a general interaction pattern $\mathcal{I}$ to that of realizing the same $f$ on a star. This transformation requires its own non-reusable correlated randomness setup (in addition to the setup for the underlying star-pattern protocol).

TheOREM 1 (INFORMAL). There exists an efficient transformation $\mathcal{T}$ that, for any n-party interaction pattern $\mathcal{I}$ and any star-compliant protocol $\Pi^{\star}$ for computing $f$, generates an $\mathcal{I}$-compliant protocol $\Pi^{\mathcal{I}}$ for computing $f$ in the nonreusable correlated randomness setup model. If $\Pi^{\star}$ is statistical/computational semi-honest secure then the resulting $\Pi^{\mathcal{I}}$ is statistical/computational malicious secure. Moreover, the randomness-size (resp. communication complexity) are only a factor of $O(n \lambda)$ (resp. $O\left(n^{2} \lambda\right)$ ) above those of the underlying protocol $\Pi^{\star}$, where $\lambda$ is a security parameter.

We remark that the above theorem is for the case where $f$ operates over binary inputs. When the function $f$ computed by $\Pi^{\mathcal{I}}$ accepts larger inputs, our transformation requires $\Pi^{\star}$ for a different (but related) function $f^{\prime}$ that operates over binary inputs. We also remark that we only consider $\mathcal{I}$ with a single sink; in the semi-honest case, this can be trivially extended to allow for multiple sink nodes.

II. Information-Theoretic Protocols for General Functions. In the information-theoretic setting, we present in
Section 3 perfectly (resp., statistically) secure protocols for computing any deterministic function against semi-honest (resp., malicious) adversaries. For $f:\{0,1\}^{n} \rightarrow\{0,1\}$, our semi-honest protocols give each party $2^{n}$ bits of correlated randomness and require each party to communicate $O\left(n \cdot 2^{n}\right)$ bits. For malicious security, the correlated randomness and communication are of size $O\left(\lambda n \cdot 2^{n}\right)$, with $\lambda$ the security parameter.

Theorem 2 (Informal). For every $f:\{0,1\}^{n} \rightarrow\{0,1\}$ and any DAG interaction pattern $\mathcal{I}$, there is a semi-honest, perfectly-secure, $\mathcal{I}$-compliant protocol for $f$, in which each party gets $2^{n}+1$ bits of correlated randomness and sends at most $n \cdot 2^{n-1}$ bits of communication. Also, there is a malicious, statistically-secure, $\mathcal{I}$-compliant protocol for $f$, in which each party gets $O\left(\lambda n \cdot 2^{n}\right)$ bits of correlated randomness and sends at most $O\left(\lambda n \cdot 2^{n}\right)$ bits of communication.

For non-DAG patterns, we can use our protocol for star (which is a special case of the above; see Section 3.2) and then apply our reduction to obtain:

Theorem 3 (Informal). For every $f:\{0,1\}^{n} \rightarrow\{0,1\}$ and any interaction pattern $\mathcal{I}$, there is a semi-honest, perfectlysecure, $\mathcal{I}$-compliant protocol for $f$, in which each party gets $n+(n+1) \cdot\left(2^{n}+1\right)$ bits of correlated randomness and sends at most $n^{2} \cdot\left(2^{n-1}+2\right)$ bits of communication. Also, there is a malicious, statistically-secure, $\mathcal{I}$-compliant protocol for $f$, in which each party gets $O\left(\lambda n \cdot 2^{n}\right)$ bits of correlated randomness and sends at most $O\left(\lambda n^{2} \cdot 2^{n}\right)$ bits of communication.

We stress again that for small input domains, these protocols could be quite practical, see Table 1 for some concrete numbers for computing general functions with binary inputs, a single output and semi-honest security.

Better Communication Complexity. For the chain interaction pattern, we describe in Section 3.1 protocols for computing arbitrary functions where the total communication complexity is only polynomial in the input size, though the correlated randomness is still exponential.

Theorem 4 (InformaL). For every $f:\{0,1\}^{n} \rightarrow\{0,1\}$, there is a semi-honest, perfectly-secure protocol for $f$ with a chain pattern in which each party gets at most $n \cdot 2^{n}$ bits of correlated randomness and sends at most $n$ bits of communication. Also, there is a malicious, statistically-secure protocol for $f$ with a chain pattern in which each party gets at most $O\left(\lambda n \cdot 2^{n}\right)$ bits of correlated randomness and sends at most $O\left(n^{2}+\lambda n\right)$ bits of communication.

In the full paper, we give evidence against the possibility of extending this result to more general interaction patterns: concretely, we show that even in a network $\mathcal{N}_{n}$ consisting of two chains (each of length $n$ ) that lead to a common endpoint, a similar protocol would imply a 3 -server protocol for information-theoretic PIR 13] with poly-logarithmic communication, which is an unexpected result.

Theorem 5 (Informal). Assume that, for every $f$ : $\{0,1\}^{2 n} \rightarrow\{0,1\}$, there exists a semi-honest, statisticallysecure $\mathcal{N}_{n}$-compliant protocol that computes $f$ with communication complexity $c(n)$. Then, there exists an (interactive, statistical) 3-server PIR protocol, with communication complexity $O(c(\log N)+\log N+\log 1 / \epsilon)$, where $N$ is the database size and $\epsilon$ is the statistical security parameter. 


\begin{tabular}{|l|c|c|}
\hline & Correlated Randomness & Online Communication \\
\hline Previous work: Star 3 & $2.5 \mathrm{MB}$ & $2.5 \mathrm{MB}$ \\
\hline Star (Lemma 3.2) & $128 \mathrm{~KB}$ & $128 \mathrm{~KB}$ \\
\hline Chain (Theorem 11) & $2.5 \mathrm{MB}$ & $20 \mathrm{bits}$ \\
\hline DAG (Theorem 2) & $128 \mathrm{~KB}$ & $1.25 \mathrm{MB}$ \\
\hline Gen. Patterns (Theorem 3) & $2.5 \mathrm{MB}$ & $25 \mathrm{MB}$ \\
\hline
\end{tabular}

Table 1: Concrete complexity numbers (per party) for $n=20$ for computing general functions with single output with information-theoretic security in the semi-honest model.

\begin{abstract}
III. Efficient Information-Theoretic Protocols for Symmetric Functions. For symmetric functions, we construct in the full paper efficient, perfectly (resp., statistically) secure protocols over a chain against semi-honest (resp., malicious) adversaries where both the offline and online phases are polynomial in the input size.
\end{abstract}

TheORem 6 (Informal). For every symmetric binary function $f:\{0,1\}^{n} \rightarrow\{0,1\}$, there is a semi-honest perfectlysecure protocol for $f$ for the chain network in which each party gets $(n+1)^{2}$ bits of correlated randomness and sends at most $(n+1)^{2}$ bits of communication. Also, there is a malicious statistically-secure protocol for $f$ for the chain in which each party gets $O\left(\lambda n^{2}\right)$ bits of correlated randomness and sends at most $O\left(\lambda n^{2}\right)$ bits of communication, where $\lambda$ is the statistical security parameter. Moreover, both these protocols have efficient simulators.

IV. Computational Protocols for General Functions from Obfuscation. In the computational setting, we observe in the full paper that the multi-input functional encryption scheme of 29 already yields a protocol for computing general functions with a star interaction against semihonest adversaries based on indistinguishability obfuscation $(i \mathrm{O})[2,24$ and one-way functions. Combining this with Theorem [1] we obtain a malicious-secure protocol for computing general functions with general interaction patterns.

Theorem 7 (Informal). Assuming $i O$ for general circuits and one-way functions, for every interaction pattern $\mathcal{I}$, there exists an $\mathcal{I}$-compliant protocol for computing any polynomial-time function that achieves malicious security against any number of corruptions, in the (non-reusable) correlated randomness setup model.

Making Correlated Randomness Reusable. We also present a generic procedure to transform any non-reusable correlated randomness setup into one that is reusable. Our transformation works in the common random string (CRS) model where the size of the CRS grows linearly with the number of uses of the correlated randomness. We note, however, that since the CRS is "public" randomness, it can be easily compressed in the random oracle model.

Our transformation builds on the recent work of [34] and inherits their assumptions of $i \mathrm{O}$ and fully homomorphic encryption (FHE). Composing our transformation with Theorem 7 , we obtain the following:

Theorem 8 (Informal). Assuming $i O$ for general circuits and FHE, for every interaction pattern $\mathcal{I}$, there exists an $\mathcal{I}$-compliant protocol for computing any polynomial-time function that achieves malicious security against any number of corruptions. The protocol uses a reusable correlated randomness setup in the CRS model where the size of the CRS grows linearly with the number of uses of the protocol.
Necessity of $i \mathbf{O}$. We note that general-purpose $i \mathrm{O}$ is a necessary assumption for the above results. Indeed, for the special case of a star pattern, it was already shown by 29 that a secure protocol for general functions implies generalpurpose $i \mathrm{O}$.

V. Computational Protocols for Symmetric Functions from Multilinear Maps. For the case of symmetric functions, we describe in the full paper a much simpler protocol for general interaction patterns that uses multilinear maps but does not require general-purpose obfuscation. The security of that protocol reduces to a very simple variant of the Multilinear Decisional Diffie-Hellman (MDDH) assumption over multilinear maps 8, 23], which we call the bookend $M D D H$ assumption. Unfortunately, this assumption (and indeed the security of our protocol) does not hold for any of the current multilinear map candidates $23,14,26,15$.

VI. Implications to standard MPC. We note that our results for MPC with general interaction patterns also have relevance to standard MPC over fully connected networks. For instance, suppose that there is a cost $c_{i, j}$ associated with sending a message from $P_{i}$ to $P_{j}$. Our results reduce the question of minimizing the total cost of an MPC protocol in this setting to a combinatorial optimization problem. For instance, for standard $n$-party MPC where only $P_{1}$ has an output, our results imply general protocols with only $2 n-2$ point-to-point messages (e.g., a chain from $P_{1}$ to $P_{n}$ and back), which can be shown to be optimal.

\subsection{Technical Overview}

We now give an overview of our protocols. We only focus on some of the main results and defer the reader to the technical sections for the details on all of our results.

Reduction to Star Pattern. Recall that our goal here is to transform an $n$-party protocol for computing general functions with a star pattern into another protocol for general interaction patterns $\mathcal{I}$. For simplicity, here we focus only on computing functions with binary inputs and achieving semi-honest security and defer the reader to Section 2 for the general case. It is instructive to consider the naive protocol where each party just sends to the evaluator whatever message it was supposed to send in the underlying star protocol over the paths in $\mathcal{I}$. This protocol falls short of providing the "best possible security" for the pattern $\mathcal{I}$, because in the star pattern the inputs of all the corrupted parties are free while some (or all) of them can be fixed in $\mathcal{I}$.

To do better, we start with the observation that in the underlying star protocol, once all the randomness is fixed, then every party $P_{i}$ sends one of two fixed messages $\left(m_{i}^{0}, m_{i}^{1}\right)$ to the evaluator, depending upon its input bit. In our transformation, we share the two possible messages of each party using an $n$-out-of- $n$ secret-sharing, giving each party one share of every message. These shares comprise the correlated ran- 
domness that each party gets under our transformation. The omission of any share will prevent the reconstruction of the original message. Thus, if only one of the two shares of party $P_{i}$ 's messages is sent by $P_{j}$ then this in a way forces $P_{i}$ 's input to be fixed, even if $P_{i}$ is corrupted.

The challenge is to let $P_{j}$ know which of the two shares to send to the evaluator without revealing $P_{i}$ 's input. To that end, we distribute the shares of $P_{i}$ 's two messages to all the parties in a random but consistent order. That is, either they all get first the share of $m_{i}^{0}$ followed by share of $m_{i}^{1}$, or vice-versa. Furthermore, this random permutation bit is given to party $P_{i}$. During the protocol $P_{i}$ will xor that bit with its input, sending the resulting bit to the other parties to indicate which shares they should send to the evaluator and which to omit. See Section 2 for more details of this protocol and its proof of security.

Information-Theoretic Protocols for General Functions. Our information-theoretic protocols follow a somewhat similar approach to the general reduction discussed above. Specifically, we secret-share the truth table of $f$, giving each party a share for each of the $2^{n}$ inputs, and then have the honest parties omit some of their shares during the protocol run. This is done so as to ensure that when the input of $P_{i}$ is fixed, the adversary can obtain all the shares for inputs with $x_{i}=0$ or all the shares for inputs with $x_{i}=1$, but not both.

We remark that this is easy to implement in a star pattern: since the only fixed inputs are those of the honest parties, we can have each honest party send to the evaluator only the shares consistent with its own input bit. A protocol for a general pattern can be obtained using the transformation from above, but we can get a better communication complexity by instead tailoring the share-omission rules to the communication pattern. To obtain security in the maliciousadversary model, we add authentication information to the correlated randomness. See Sections 3.1 and 3.2 for details.

Efficient Information-Theoretic Protocols for Symmetric Functions. In the case of symmetric functions, we capitalize on the fact that there is a small representation of the truth table of the function. In particular, the residual function in this representation can be obtained from the global truth table by having each party locally drop one of the rows, depending on its input.

A similar approach was considered by 33 in the computational setting. In their case, the rows of the truth table were encrypted using an additively homomorphic scheme so that party $P_{i+1}$ does not learn what row was dropped by party $P_{i}$. In our information-theoretic setting, however, such an additively homomorphic scheme is not available so we use a different hiding mechanism. Specifically, we view messages as matrices, letting each party in the protocol multiply its received message by a random matrix (which is given to it in its correlated randomness), then dropping one column and forwarding the result to the next party. The correlated randomness of the evaluator consists of the columns of the resulting product matrix, tagged by the function output and permuted randomly. In a run of the protocol, the evaluator will receive one of these columns and will use it to determine the corresponding function output.

Adding security against a malicious adversary is harder here than in our other information-theoretic protocols, since parties do more than just forwarding some pre-determined messages that are given to them as part of their correlated randomness. Our solution still uses authentication to force the corrupted parties to send the right messages, but we must ensure that the added authentication information does not leak information on potential messages that are not sent in a particular run of the protocol. To ensure that, we use an authentication mechanism that doubles also as a randomness extractor, namely the "extractor-MAC" construction from [18], which is based on almost-universal hashing [42]. We also need to withhold from the evaluator the columns of the product matrix, replacing them by just the authentication information needed to recognize these columns. Details and proofs of security in the full paper.

Computational Protocols for General Functions. Here, we focus on our generic transformation from a non-reusable correlated randomness setup $\mathcal{C} \mathcal{R}_{\text {nr }}$ into a reusable one $\mathcal{C} \mathcal{R}$. As mentioned earlier, our transformation works in the CRS model.

Our starting idea is to use an MPC protocol $\Pi$ to compute the function $F_{\text {cr }}$ that takes small randomness as input and outputs $L=\operatorname{poly}(k)$ independent instances of $\mathcal{C} \mathcal{R}_{\mathrm{nr}}$. Note that such a function $F_{\text {cr }}$ is easy to define: on input a short random seed, $F_{\text {cr }}$ first expands it into a large pseudorandom string using a PRG (of appropriate stretch) and then uses different "chunks" of the resultant string to compute $L$ independent instances of $\mathcal{C R}_{\mathrm{nr}}$. Now, fix an honest execution of $\Pi$ and consider the view view $i$ of each party $i$ in the execution consisting of its random tape and the protocol messages. Note that if each $\mid$ view $_{i} \mid$ was independent of $L$, then we could simply set $\left(\right.$ view $_{1}, \ldots$, view $\left._{n}\right)$ as an instance of the reusable correlated randomness setup $\mathcal{C} \mathcal{R}$. This is because given view $_{i}$, party $P_{i}$ can locally compute the output of $\Pi$, which consists of $L$ instances of $\mathcal{C} \mathcal{R}_{\mathrm{nr}}$.

Thus, we have effectively reduced our problem of making any correlated randomness setup reusable to the problem of constructing an MPC protocol $\Pi_{\text {out-ind }}$ that computes $n$ party functions with "long" outputs where the communication complexity of the protocol as well as the size of randomness of each party is independent of the function output length. A moments reflection, however, reveals that such a protocol is impossible in the standard model ${ }^{2}$ Instead, our solution will use a (long) common random string (CRS).

Our starting point is a recent work of Hubáček and Wichs 34], who constructs a secure two-party computation protocol where the communication complexity of the protocol is independent of the function output length. However, the size of the randomness of each party does grow with the function output length. We extend their protocol to the multiparty setting. Let $\Pi_{\text {long-rand }}$ denote the resulting protocol. Our key observation then is that the long randomness of the parties in $\Pi_{\text {long-rand }}$ can be "compressed" by using a long public CRS. In particular, we transform $\Pi_{\text {long-rand }}$ into a new protocol $\Pi_{\text {short-rand }}$ using a public random string CRS $=\mathrm{CRS}_{1}, \ldots, \mathrm{CRS}_{n}$ where each $\mathrm{CRS}_{i}$ is as long as the function output length. The randomness of each party $P_{i}$ in $\Pi_{\text {short-rand }}$ is set to be a short seed $r_{i}$. At the start of the protocol, $P_{i}$ first locally computes a large random string $R_{i}=\operatorname{PRG}\left(r_{i}\right) \oplus \mathrm{CRS}_{i}$. It then executes the strategy of the $i$ 'th party in $\Pi_{\text {long-rand }}$ using $R_{i}$ for the rest of the protocol.

Combining the above steps, we obtain our desired MPC protocol. We stress that we are able to bypass the aforemen-

\footnotetext{
${ }^{2}$ Consider an execution of $\Pi_{\text {out-ind }}$ for evaluating a PRG with "long" stretch. The view of any party $i$ in the protocol is a "compressed" representation of the long protocol output. This can be used to derive a computational incompressibility argument, similar to several recent works [1, 10, 34.
} 
tioned impossibility result since we are working in the CRS model, where the size of the CRS grows with the function output length.

Computational Protocols for Symmetric Functions. For symmetric functions, we use multilinear maps to construct simple protocols that achieve computational security against semi-honest adversaries. Here we focus on the star pattern; a protocol for general interaction patterns can be obtained by composing the star protocol with our general reduction to star.

Consider an $n$-level multilinear map where $[x]_{i}$ denotes an encoding of $x$ at level $i$. In our star protocol, as part of the correlated randomness, each party $P_{i}$ is given two level-1 encodings $\left[a_{i}\right]_{1},\left[a_{i} \times r\right]_{1}$ for random and independent elements $a_{i}$ 's and the same random $r$. Let $A=\prod_{i=1}^{n} a_{i}$ denote the product of the $a_{i}$ 's. The evaluator is given of all the $n+1$ (level- $n$ ) encodings $\left[b_{i}\right]_{n}=\left[A \times r^{i}\right], i=0,1, \ldots n$ in a random order, where each $b_{i}$ is tagged with the function value $f_{i}=f\left(1^{i} 0^{n-i}\right)$.

In the protocol, each party $P_{i}$ simply sends the encoding $\left[a_{i}\right]_{1}$ or $\left[a_{i} \times r\right]_{1}$, depending upon whether its input bit is 0 or 1 , respectively. The evaluator multiplies the $n$ encodings that it receives and then compares the resulting encoding against the $b_{i}$ 's to determine the function output. More details are provided in the full version. As mentioned above, this protocol is unfortunately insecure using current multilinear-map candidates.

\subsection{Related Work}

There is a very large body of recent work about minimizing interaction in cryptography (mostly concentrating on the number of rounds, rather than the number of messages): whereas classical MPC results show that every cryptographic task can be realized with sufficient interaction, popular recent research topics such as garbling schemes 44 , 4. 30, fully homomorphic encryption 25, functional encryption 40, 7, 39, and obfuscation 2, 24], are all about minimizing interaction. Our work can be seen as taking the question of "what can we do with a given type of interaction" to its ultimate level of generality. One can view the previous notions, as well as standard interactive MPC, as special cases of this general problem.

Another large body of work, originating from [19], studies the problem of secure communication (or message transmission) in general networks, where only certain pairs of parties can communicate with each other. This goal is trivialized when allowing a correlated randomness set-up, as we do here, and so the challenges that arise in that setting are very different from the ones we face in our work. The same is true for a recent extension of this problem to secret sharing in general networks [41, a task whose feasibility reduces to that of secure message transmission.

Another line of work, originating from earlier works in the context of distributed computing [20], studies the possibility of realizing MPC on sparse networks 22, 11. These works use specially designed (expander-based) networks to allow MPC in graphs of a small degree. To this end, they also need to relax the traditional goal of MPC by assuming that some honest inputs are being compromised. However, in contrast to the model considered in our work, being compromised there means "known or fixed by the adversary" as opposed to being "free" in the sense considered here. In particular, general solutions for MPC in that model have no consequences for obfuscation.
Finally, Kearns et al. 36. also study secure computation in a model with restricted communication. Their restriction is more liberal than our definition of interaction pattern: each message from $P_{i}$ to $P_{j}$ should be computed by a small neighborhood of $P_{i}, P_{j}$ (in an undirected network graph). Their positive results provide computational security against a single corrupted party, a limitation which they show to be inherent to their model. In contrast, our protocols provide a meaningful notion of security with respect to any number of corrupted parties.

\section{A REDUCTION TO STAR}

In the Technical Overview (Section 1.3) we described a reduction from the problem of realizing a function $f$ using an arbitrary interaction pattern $\mathcal{I}$ to that of realizing the same $f$ on a star. Our transformation is informationtheoretic and does not require any cryptographic assumptions, but it requires its own non-reusable correlated randomness (in addition to whatever setup is needed for the underlying star-pattern protocol). Below we prove that if the underlying star protocol is semi-honest secure then so is the resulting general-pattern protocol, achieving possibly even better security. Furthermore, in Section 2.2 we modify the transformation so that the general-pattern protocol is malicious-secure. This proof will imply that the star interaction pattern is complete for secure computations with restricted interaction patterns.

Our transformation assumes that the function $f$ depends on all its inputs, that it has only a single party with output (as this is inherent in having a secure star-protocol for $f$ ), and that the interaction pattern has at least one message path from every party to the evaluator. We note that in the semi-honest model one can easily extend a solution for the one output case to multiple outputs by just running separate protocols for the different outputs (piggybacking over the same messages of $\mathcal{I}$ as needed).

\subsection{The Semi-Honest Transformation}

THEOREM 9. There exists an efficient transformation $\mathcal{T}$ such that for any function $f:\{0,1\}^{n} \rightarrow\{0,1\}$ that depends on all inputs, any interaction pattern $\mathcal{I}$ with a single sink, and any star-compliant protocol $\Pi^{\star}$ for $f, \mathcal{T}\left(\Pi^{\star}, \mathcal{I}\right)$ generates an $\mathcal{I}$-compliant protocol $\Pi^{\mathcal{I}}$ for $f$ (with non-reusable correlated randomness) with the following properties:

- If in $\Pi^{\star}$ each party gets at most $R$ bits of correlated randomness and sends at most $M$ bits of communication, then in $\Pi^{\mathcal{I}}$ each party gets at most $R+2 n \cdot M$ bits of correlated randomness and sends at most $n^{2}(M+1)$ bits of communication.

- If $\Pi^{\star}$ is perfect/statistical/computational semi-honest secure then so is the resulting $\Pi^{\mathcal{I}}$.

Proof. Let $P_{1}, P_{2}, \ldots, P_{n+1}$ be the parties, and assume without loss of generality that $P_{n+1}$ is the evaluator. Let $\Pi^{\star}=\left(G^{\star}{ }^{\star}\right.$, Msg.int ${ }^{\star}$, Msg.eval $\left.{ }^{\star}\right)$ be a protocol for computing $f$ on the star, where Gen ${ }^{\star}$ generates correlated randomness, Msg.int ${ }^{\star}$ is the next message function of the parties, and Msg.eval ${ }^{\star}$ is used by the evaluator to compute the output. For any communication pattern $\mathcal{I}$, we construct a protocol $\Pi^{\mathcal{I}}=\left(\mathrm{Gen}^{\mathcal{I}}\right.$, Msg.int ${ }^{\mathcal{I}}$, Msg.eval $\left.{ }^{\mathcal{I}}\right)$ for computing $f$ on $\mathcal{I}$ as follows.

Setup. The randomness-generation procedure $\mathrm{Gen}^{\mathcal{I}}$ begins by running Gen ${ }^{\star}$ to generate correlated randomness 
$r_{1}, \ldots, r_{n+1}$ for the underlying star protocol, and then proceeds as follows:

1. For each party $i \leq n$, and for every input bit $\sigma \in$ $\{0,1\}$, compute a message that $P_{i}$ could send in the underlying star protocol, $m_{i}^{\sigma} \leftarrow$ Msg.int ${ }^{\star}\left(\sigma, r_{i}\right)$.

2. Compute an $n$-out-of- $n$ secret sharing of each message $m_{i}^{\sigma}$. Let $m_{i, 1}^{\sigma}, \ldots, m_{i, n}^{\sigma}$ denote the $n$ shares of $m_{i}^{\sigma}$.

3. Choose random permutation bits $b_{1}, \ldots, b_{n}$, one for each party $P_{i}, i \leq n$.

The correlated randomness of each party $P_{i}$ includes the permutation bit $b_{i}$, and all the pairs $\left(m_{j, i}^{b_{j}}, m_{j, i}^{1-b_{j}}\right)$ for every $j \leq n$. Below we denote $\left(M_{j, i}^{0}, M_{j, i}^{1}\right)=\left(m_{j, i}^{b_{j}}, m_{j, i}^{1-b_{j}}\right)$. The evaluator receives in addition also $r_{n+1}$.

Messages and Output. On input $x_{i}$, each party $P_{i}$ computes $c_{i}=x_{i} \oplus b_{i}$, which determines which shares of $P_{i}$ 's messages should be used. Then it proceeds as follows:

1. $P_{i}$ sends the bit $c_{i}$ on every path to the evaluator in $\mathcal{I}$.

2. Then, for every $P_{j}$ such that some path from $P_{j}$ to the evaluator goes through $P_{i}$, party $P_{i}$ waits until it receives the bit $c_{j}$ and then sends $M_{j, i}^{c_{j}}=m_{j, i}^{x_{i}}$ on the path to the evaluator.

3. For every $P_{j}$ such that no path from $P_{j}$ to the evaluator goes through $P_{i}$, party $P_{i}$ sends both shares on some $\mathcal{I}$-path to the evaluator.

4. In addition, $P_{i}$ forwards every messages that it receives from other parties on some $\mathcal{I}$-path to the evaluator.

After receiving all the messages, the evaluator collects the set of shares $\left\{M_{i, 1}^{c_{i}}, \ldots, M_{i, n}^{c_{i}}\right\}_{i \in[n]}$ and reconstructs all the messages $m_{i}^{x_{i}}$ from the corresponding $n$ shares, for every $i \in[n]$. Finally, it runs the evaluator algorithm Msg.eval ${ }^{\star}$ on inputs $r_{n+1}$ and $\left\{m_{i}^{x_{i}}\right\}_{i \in[n]}$ and returns its output.

This completes the description of $\Pi^{\mathcal{I}}$. The correctness of the protocol is easy to verify.

Complexity. To achieve the communication complexity stated in Theorem 9 the parties need to send their messages only once on all paths to the evaluator and forward each message that they receive only once. Specifically each $P_{i}$ needs to send the bits $c_{j}$ (either its own or others') toward each $P_{j^{\prime}}$ downstream only in the first opportunity that it has according to $\mathcal{I}$. Similarly it needs to send shares toward the evaluator (both its own and forwarded) only in the last opportunity that it has according to $\mathcal{I}$. All other $\mathcal{I}$ messages (if any) should be empty. Done this way, the complexity of the resulting $\Pi^{\mathcal{I}}$ depends only on the number of parties $n$ and NOT on the number of messages $N$ in the communication pattern $\mathcal{I}$.

Some further optimization is possible, in that each $P_{i}$ need not forward all messages from other parties, it can drop messages that it already knows are inconsistent with the $c_{j}$ 's that it saw. This modification changes the (worst-case) complexity stated in Theorem 9 by at most a small constant factor.

Proof of Security. We need to describe a simulator $\operatorname{Sim}^{\mathcal{I}}$ for the resulting protocol $\Pi^{\mathcal{I}}$, using the simulator $\operatorname{Sim}^{\star}$ of the underlying star-protocol. The simulator, $\operatorname{Sim}^{\mathcal{I}}$, gets the corrupted parties' input and the residual truth table, and needs to produce the correlated randomness of the corrupted parties and the messages of the honest parties. It will utilize the simulator of the star to achieve this goal. As explained above, the residual truth table that $\operatorname{Sim}^{\mathcal{I}}$ gets is more restricted than that of $\mathrm{Sim}^{\star}$, as in $\mathcal{I}$ some of the corrupt parties' inputs may be fixed. However, we show that this residual function is sufficient to simulate the communications. Let $T \subseteq[n+1]$ be the set of corrupted parties in a run of $\Pi^{\mathcal{I}}$, and partition it into fixed and free parties $T=T_{\text {Fixed }} \cup T_{\text {Free }}$. Denote the set of honest parties by $H=[n+1] \backslash T$. Let $T^{*}=T_{\text {Free }}$ be the set of corrupted parties used for simulator $\mathrm{Sim}^{\star}$ and $H^{*}=T_{\text {Fixed }} \cup H$ be the set of honest parties.

The $\mathcal{I}$-simulator $\operatorname{Sim}^{\mathcal{I}}$ gets the input bits $x_{i}$ for all $i \in$ $T$ and the residual function $f^{\prime}\left(x_{T_{\text {Free }}}\right)=f\left(x_{H^{*}}, x_{T_{\text {Free }}}\right)$. It runs the star-simulator $\operatorname{Sim}^{\star}$, giving it the input bits $x_{i}$ for $i \in T_{\text {Free }}$ and the same residual function. Sim ${ }^{\star}$ returns the correlated randomness $r_{i}$ for $i \in T_{\text {Free }}$ and messages $m_{i}$ for $i \in H^{*}$. (Recall that $m_{i}=m_{i}^{x_{i}}$ for some $x_{i} \in\{0,1\}$, but $\operatorname{Sim}^{\mathcal{I}}$ does not know $x_{i}$.)

Next $\operatorname{Sim}^{\mathcal{I}}$ computes the corrupted-party messages of the underlying star protocol as $m_{i}^{\sigma} \leftarrow \operatorname{Msg.int}{ }^{\star}\left(\sigma, r_{i}\right)$ for $i \in$ $T_{\text {Free }}, \sigma \in\{0,1\}$, and also chooses random bits $b_{1}, \ldots, b_{n}$. It computes $n$-out-of- $n$ secret sharing of all the $m$ 's that it knows, chooses at random shares for the $m$ 's that it does not know, and orders the shares as follows:

- For all $i \in T_{\text {Free }}, j \in[n]$, the simulator sets $c_{i}:=b_{i} \oplus x_{i}$ and then for $\sigma \in\{0,1\}$ it sets $M_{i, j}^{\sigma}$ to be the $j$ 'th share of the message $m_{i}^{\sigma \oplus b_{i}}$.

- For all $i \in H^{*}, j \in[n+1]$, the simulator sets $c_{i}:=b_{i}$ and then it sets $M_{i, j}^{b_{i}}$ to be the $j$ 'th share of the message $m_{i}$, and it chooses $M_{i, j}^{1-b_{i}}$ uniformly at random.

The $\mathcal{I}$-simulator $\operatorname{Sim}^{\mathcal{I}}$ gives every $i \in T$ the correlated randomness $b_{i}$ and shares $\left\{\left(M_{j, i}^{0}, M_{j, i}^{1}\right): j \in[n]\right\}$, and if the evaluator is corrupted then $\operatorname{Sim}^{\mathcal{I}}$ gives it also the correlated randomness $r_{n+1}$ of the underlying star protocol. Finally. $\mathrm{Sim}^{\mathcal{I}}$ runs the actual protocol $\Pi^{\mathcal{I}}$ using the shares that it computed and the $c_{i}$ bits to determine the honest parties' messages in the $\mathcal{I}$-protocol.

We observe that the simulated view is identical/statistiallyclose/computationally indistinguishable to the real view, depending on the properties of the underlying simulator Sim ${ }^{\star}$, Indeed, if the simulated view contains all the shares of some messages $m$ of the underlying protocol, then either $m$ was produced directly by $\mathrm{Sim}^{\star}$ or it was computed from the correlated randomness $r_{i}$ produced by $\mathrm{Sim}^{\star}$. All other shares in the view, as well as the permutation bits $b_{i}$, are uniformly random and independent of everything else.

Handling Functions Over a Large Domain. The transformation above can handle a function with a large range without any change, but its efficiency relies crucially on the domain being small, so that once the correlated randomness is fixed each party has only a small number of messages that it can possibly send in the star protocol, depending on its input. Applying the transformation as-is to a function $f: D^{n} \rightarrow R$ with a large domain $D$, would increase the complexity of the star protocol for $f$ roughly by a factor of $|D|$.

To do better, we can always represent each inputs to $f$ in binary using $\ell=\log |D|$ bits and apply the above 
transformation to a star protocol for the modified function $f^{\prime}:\{0,1\}^{n \ell} \rightarrow R$, defined as:

$$
f^{\prime}\left(x_{1}, \ldots, x_{\ell n}\right)=f\left(\left(x_{1}, \ldots, x_{\ell}\right), \ldots,\left(x_{(n-1) \ell+1}, \ldots, x_{n \ell}\right)\right) .
$$

Note that this requires that we view the $n$-player interaction pattern $\mathcal{I}$ as an $n \ell$-player pattern, which we can always do by introducing dummy communication flows between the virtual players that are implemented by a single real player. More importantly, though, it requires a star protocol for $f^{\prime}$ rather than a star protocol for $f$.

\subsection{Reduction for Malicious Security}

THEOREM 10. There exists an efficient transformation $\mathcal{T}$ such that for any function $f:\{0,1\}^{n} \rightarrow\{0,1\}$ that depend on all its inputs, any $n$-party interaction pattern $\mathcal{I}$ with a single sink, and any star-compliant protocol $\Pi^{\star}$ for $f$, $\mathcal{T}\left(\Pi^{\star}, \mathcal{I}\right)$ generates an $\mathcal{I}$-compliant protocol $\Pi^{\mathcal{I}}$ for $f$ (with non-reusable correlated randomness) with the following properties:

- If in $\Pi^{\star}$ the evaluator gets $R$ bits of correlated randomness and the other parties send at most $M$ bits of communication each, then in $\Pi^{\mathcal{I}}$ each party gets at most $R+2 n \cdot M \cdot O(\lambda)$ bits of correlated randomness and sends at most $n R+n^{2} M \cdot O(\lambda)$ bits of communication, with $\lambda$ the statistical security parameter.

- If the underlying protocol $\Pi^{\star}$ is statistical/computational semi-honest secure, then the resulting $\Pi^{\mathcal{I}}$ is statistical/computational malicious secure.

Proof. (sketch) The transformation for malicious security is very similar to the semi-honest transformation from above, except that we also secret-share the evaluator randomness $r_{n+1}$, and we authenticate all the messages (say, using an information-theoretic two-time MAC, e.g. 3-wise independent hash functions). Specifically, the parties are given the authentication tags for the messages that they may need to send (and they attach these tags to the messages that they actually send), and the evaluator is given the keys to verify these MACs.

The complexity is easy to verify, and the security proof is quite similar to the one from above. The main difference is that before $\operatorname{Sim}^{\mathcal{I}}$ learns the inputs of the fixed corrupted parties, it needs to give all the corrupted parties their correlated randomness and the messages from honest parties (other than the last one). Moreover, $\operatorname{Sim}^{\mathcal{I}}$ needs to extract these inputs from messages that the fixed corrupted parties send.

To do this, $\operatorname{Sim}^{\mathcal{I}}$ chooses the bits $b_{i}$ and the shares uniformly at random (and the authentication keys and tags are chosen as in the protocol). When a fixed corrupted party $P_{i}$ sends the bit $c_{i}$ towards an honest party, $\operatorname{Sim}^{\mathcal{I}}$ extracts the input bit $x_{i}=c_{i} \oplus b_{i}$. Once it has all the input bits $x_{i}$ for the fixed corrupted parties, $\operatorname{Sim}^{\mathcal{I}}$ gets the residual function and it can then run the semi-honest simulator Sim ${ }^{\star}$ for the underlying star protocol (choosing arbitrary inputs for the free corrupted parties). Now Sim ${ }^{\mathcal{I}}$ learns from Sim ${ }^{\star}$ the correlated randomness $r_{i}$ for corrupted parties and the messages $m_{i}$ for the honest parties, so it can compute all the relevant $m_{i}$ 's and choose the shares of the last honest party to match these $m_{i}$ 's (and also the randomness $r_{n+1}$ of the evaluator, if it is corrupted). Finally $\operatorname{Sim}^{\mathcal{I}}$ can compute the authentication tags using the keys that it prepared for the evaluator, so it has everything that it needs for the simulation.
Another difference from the semi-honest case is that, when the evaluator is honest, we need to use the authentication tags. Namely, if the evaluator receives a message which is not consistent with the shares that $\operatorname{Sim}^{\mathcal{I}}$ generated, then the simulator aborts, since this is what would happen whp in the protocol itself.

It is not hard to see that this simulation strategy produces a distribution which is statistically close (upto authentication error) to the real-protocol distribution if $\mathrm{Sim}^{\star}$ is perfect or statistical, and is computationally indistinguishable from the real-protocol distribution if $\mathrm{Sim}^{\star}$ is computational.

\section{INFO-THEORETIC PROTOCOLS FOR GENERAL FUNCTIONS}

Below, we present information-theoretically secure protocols with one-time setup for computing arbitrary functions. We begin in Section 3.1 with a protocol for computing arbitrary functions on a chain which is communication efficient (but still requires exponential randomness). Then, in Section 3.2, we describe a protocol for computing arbitrary deterministic functions on arbitrary DAGs with exponential communication and randomness. We also describe, in Section 3.2.1. a protocol which is insecure as per our definition, but has some interesting implications for garbled circuits.

We identify an $n$-input function $f\left(x_{1}, \ldots, x_{n}\right)$ with a binary decision tree for $f$. Each input is associated with a level in the tree and the input ordering in the tree is made to respect the topological order in the DAG. That is, if there is a path from party $A$ to $B$ in the DAG, then we put the input of $A$ before the input of $B$ in the decision tree. In particular, for a chain network, the ordering in the tree agrees completely with the linear order of nodes on the chain.

In the protocols, a party associated with level- $i$ in the tree will be given correlated randomness associated with each edge leading from level $i$ to level $i+1$. During the computation of the function it will send some of the information which is associated with the edges that match its input (i.e. left edges if the input is 0 and right-edges if it is 1). This can be visualized as each party marking some of the edges in its layer of the tree. The markings create a single marked path from the root to a leaf. The value of the function will be computed by the evaluator based on the information in this leaf.

In the description below, we use the following notations: We have a height- $n$ decision tree $\mathcal{T}$, with the root at level 0 and the leaves at level $n$. The left edge of every intermediate node is labeled with 0 , and the right edge is labeled with 1 . We name each node in the tree by the labels on the path leading to it, so the root is named $\epsilon$, its left- and right-children are 0 and 1 , respectively, their children are $00,01,10,11$, etc. (In the protocols we will attach labels to nodes that may be different from their names, but it is convenient to have the names fixed.) Party $P_{i}$ (who gets the $i$ 'th input bit $x_{i}$ ) is associated with level $i$ in the tree ${ }^{3}$ and a special party $\mathcal{E}$ (the evaluator) associated with the leaves. The evaluator does not have an input, but it is the one who will learn the value of the function.

\subsection{Computing Any Function on a Chain}

We consider below only a chain network, and describe communication-efficient protocols that still require exponential randomness.

\footnotetext{
${ }^{3}$ For ease of description we start the enumeration of the parties at 0 rather than 1 .
} 
THEOREM 11. For every function $f:\{0,1\}^{n} \rightarrow\{0,1\}$, there is a semi-honest, perfectly-secure protocol for $f$ for the chain network in which each party gets at most $n \cdot 2^{n}$ bits of correlated randomness and sends at most $n$ bits of communication.

Setup and Correlated Randomness. We have parties $P_{0}, \ldots, P_{n-1}, P_{n}$ who are connected in a chain starting at $P_{0}$, where the last party $P_{n}$ is the evaluator who does not have an input. Party $P_{i}$ is associated with level $i$ in the decision tree $\mathcal{T}$ for $f$.

The correlated randomness in the protocol is determined by a set of random permutations, one for every level in the tree. For level $i$ (with $2^{i}$ nodes) we select a random permutation $\pi_{i}:\{0,1\}^{i} \rightarrow\{0,1\}^{i}$, and assign to each node with name $x \in\{0,1\}^{i}$ the label $a=\pi_{i}(x){ }^{4}$ These nodepermutations induce 1-1 mappings as follows: for a node named $x \in\{0,1\}^{i}$, its label $a=\pi_{i}(x) \in\{0,1\}^{i}$ and an edge labeled $b \in\{0,1\}$ that leads to its child $(x \mid b)$, we have the mapping $m_{i}:\{0,1\}^{i} \times\{0,1\} \rightarrow\{0,1\}^{i+1}$, defined as

$$
m_{i}(a, b)=\pi_{i+1}\left(\pi_{i}^{-1}(a), b\right) .
$$

Party $P_{i}$ is given the mapping $m_{i}$ from above. That is, for the two edges $x \rightarrow(x \mid 0),(x \mid 1)$, from any node $x$ at level $i$ to its two children at level $i+1$, the party $P_{i}$ is given the tuple $\left(\pi_{i}(x), \pi_{i+1}(x \mid 0), \pi_{i+1}(x \mid 1)\right)$. These tuples are given to $P_{i}$ in an order that does not reveal any extra information about the permutations $\pi_{i}$ and $\pi_{i+1}$ (e.g., in lexicographic order of the $\pi_{i}(x)$ 's). For example, the first party $P_{0}$ is given just a single tuple, $\left(\epsilon, \pi_{1}(0), \pi_{1}(1)\right)$, and the second party $P_{1}$ is given 2 tuples, $\left(\left(0, \pi_{2}\left(\pi_{1}^{-1}(0) \mid 0\right), \pi_{2}\left(\pi_{1}^{-1}(0) \mid 1\right)\right)\right.$ and $\left.\left(1, \pi_{2}\left(\pi_{1}^{-1}(1) \mid 0\right), \pi_{2}\left(\pi_{1}^{-1}(1) \mid 1\right)\right)\right)$. Overall, $P_{i}$ is given $2^{i}$ such tuples (which takes $(i+1) \cdot 2^{i+1}$ bits to write down).

The evaluator, $\mathcal{E}$ is given the "translation" of $\pi_{n}$ to the function values, i.e., $\pi_{n}(x) \mapsto f(x)$ for all $x$. In other words, the evaluator is given the table $\left\{\left\langle a: f\left(\pi_{n}^{-1}(a)\right)\right\rangle \mid a \in\{0,1\}^{n}\right\}$, ordered lexicographically by $a$.

An illustration of the permutations and associated function values for a simple 2-input function is given in Figure 1 .

Messages in the Chain Protocol. Party $P_{i}$ with input bit $b_{i}$ gets a message $a_{i}$ from its predecessor in the chain $P_{i-1}$ (initially $a_{0}=\epsilon$ ). It applies its mapping to compute $a_{i+1} \leftarrow m_{i}\left(a_{i}, b_{i}\right)$ and sends $a_{i+1}$ to the next party $P_{i+1}$. At the end of the chain, the evaluator $\mathcal{E}$ receives $a_{n}$ and outputs the corresponding $w_{n}=f\left(\pi_{n}^{-1}\left(a_{n}\right)\right)$ from its table.

LEMMA 3.1. For any n-bit-input function $f$, the above chain-compliant protocol for computing $f$ is semi-honest secure.

Proof. Correctness is obvious. For security, let the last honest party be $P_{t}$, at level $t$ of the tree. The simulator has the inputs of all the corrupted parties, and the residual truth table of the function, i.e. for all $e_{t+1}, \ldots, e_{n-1} \in\{0,1\}^{n-t-1}$ it gets the values $f\left(b_{0}, b_{1}, \ldots, b_{t}, e_{t+1}, \ldots, e_{n-1}\right)$ where $b_{i}$ is the fixed input for $0 \leq i \leq t$.

The simulator chooses at random a sequence $a_{1}, \ldots, a_{t+1}$. For every party $P_{i}$, it fixes a mapping $m_{i}$ such that $m_{i}\left(a_{i}, b_{i}\right)=$

\footnotetext{
${ }^{4}$ Some of our techniques have superficial similarity to 38 ; in particular, the use of decision trees and permuting the nodes at each level. However, the setting and goals, as well as the technical details, are very different. In particular, they deal with two-party protocols that have unlimited interaction and they cannot provide information-theoretic security (even with correlated randomness).
}

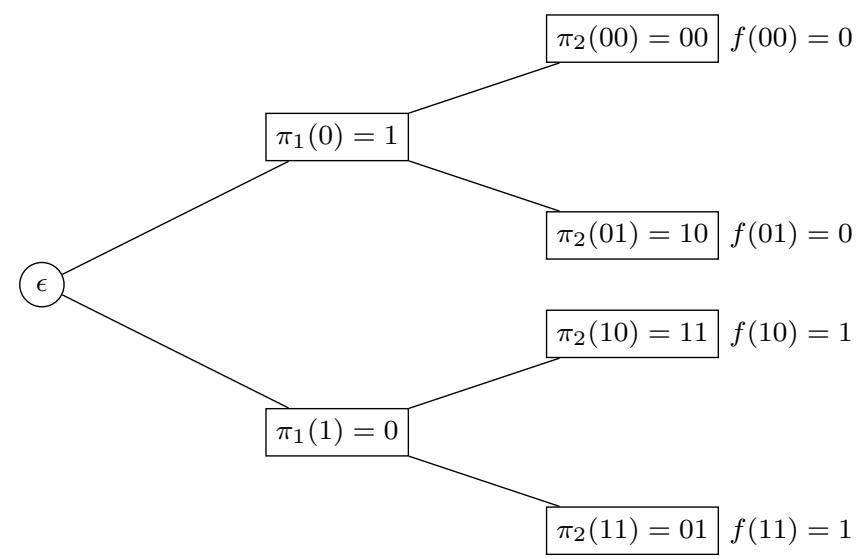

Figure 1: Correlated randomness in the chain protocol. Party $i$ gets the edges from level $i$ to $i+1$, in the form $\left\{\left(\pi_{i}(x): \pi_{i+1}(x 0), \pi_{i+1}(x 1)\right) \mid x \in\{0,1\}^{i}\right\}$, ordered lexicographically by $\pi_{i}(x)$. The evaluator $\mathcal{E}$ gets the leaves in the form $\left\{\left(\pi_{n}(x): f(x)\right) \mid x \in\{0,1\}^{n}\right\}$, ordered lexicographically by $\pi_{n}(x)$.

$a_{i+1}$, for all faulty parties, and $m_{i}\left(a_{i}, 0\right)=a_{i+1}$, for all honest parties. All other values are chosen at random. If $P_{0}$ is faulty it fixes $m_{0}\left(\epsilon, b_{0}\right)=a_{1}$ and if $P_{0}$ is honest $m_{0}(\epsilon, 0)=$ $a_{1}$. Furthermore, it chooses random mappings for all faulty parties $P_{t+1}, \ldots, P_{n-1}$. The simulator creates the table for $\mathcal{E}$ in the following manner. For $e_{t+1}, \ldots, e_{n-1} \in\{0,1\}^{n-t-1}$ it computes the chain $a_{t+2}=m_{t+1}\left(a_{t+1}, e_{t+1}\right)$ and continues $a_{i+1}=m_{i}\left(a_{i}, e_{i}\right)$ until $a_{n}$. It sets the value of the table in location $a_{n}$ to be the value of the function which it received for $e_{t+1}, \ldots, e_{n-1}$. The simulator gives the adversary the mappings for the faulty parties, the table for $\mathcal{E}$ and the sequence $a_{1}, \ldots, a_{t+1}$.

In the real execution, if the input of the honest party is 0 , then it uses the mapping created by the simulator. If the input is 1 , and say that $m_{i}\left(a_{i}, 1\right)=a_{i+1}^{\prime}$ then it changes the mapping by switching the two values $a_{i+1}$ and $a_{i+1}^{\prime}$. This results in an execution which is identical to the view of the adversary.

Malicious Security. In the full paper we convert the protocol from Section 3.1 to the malicious-adversary model, simply by authenticating the randomness, achieving the following theorem.

TheOREM 12. For every function $f:\{0,1\}^{n} \rightarrow\{0,1\}$, there is a malicious, statistically-secure protocol for $f$ for the chain network in which each party gets $O\left(\lambda n \cdot 2^{n}\right)$ bits of correlated randomness and sends $O\left(n^{2}+\lambda n\right)$ bits of communication.

\subsection{Protocol for General DAGs}

Below, we describe a protocol for computing any deterministic function on any DAG network.

ThEOREM 13. For every function $f:\{0,1\}^{n} \rightarrow\{0,1\}$ and any DAG interaction pattern $\mathcal{I}$, there is a semi-honest, $\mathcal{I}$-compliant, perfectly-secure protocol for $f$, in which each party gets $2^{n}+1$ bits of correlated randomness and sends at most $n \cdot 2^{n-1}$ bits of communication. 
To simplify the presentation, we first describe the protocol for a star network where parties just send one message to the evaluator, and later explain how to generalize. The solution has both communication and randomness complexity that are exponential in the number of parties. In the full paper, we give evidence that achieving a solution with polynomial communication might be impossible.

Recall that we identify a function $f$ with its binary decision tree, where in the case of a star network the order of inputs is arbitrary and we denote by $P_{i}$ the party associated with level $i$ in the tree, and its input is denoted $b_{i}$.

\subsubsection{A First Attempt}

We begin with a protocol which is insecure as per our definition, but will nonetheless be useful to provide some intuition. In this protocol, every party $P_{i}$ is given an inputmasking random bit $r_{i}$ and also random output-masking bits $s_{e}^{i}$ for every edge $e$ leading from level $i$ to level $i+1$ in the tree. Roughly speaking, the input-masking bits will be used to map the input string $b_{0}, \ldots, b_{n-1}$ to the root-to-leaf path $b_{0} \oplus r_{0}, \ldots, b_{n-1} \oplus r_{n-1}$ in the tree, and the output-masking bits on the edges of this path will be used to mask the function value that the evaluator gets for that leaf. Namely, the evaluator $\mathcal{E}$ is given a table that holds, for each leaf with name $c_{0}, \ldots, c_{n-1}$, the value $f\left(c_{0} \oplus r_{0}, \ldots, c_{n-1} \oplus r_{n-1}\right) \oplus$ $s_{c_{0}}^{0} \oplus s_{c_{0}, c_{1}}^{1} \oplus \cdots \oplus s_{c_{0}, \cdots, c_{n-1}}^{n-1}$.

During the protocol execution, party $P_{i}$ computes $c_{i} \leftarrow$ $b_{i} \oplus r_{i}$ and sends to the evaluator the bit $c_{i}$ and all the output masking bits associated with edges that are consistent with $c_{i}$. Namely, the bits $s_{e \mid c_{i}}^{i}$ for all $e \in\{0,1\}^{i}$. The bits $c_{0}, \ldots, c_{n-1}$ that the evaluator $\mathcal{E}$ receives define a complete root-to-leaf path in the tree. Moreover the evaluator gets the associated output-masking bits $s_{c_{0}, \ldots, c_{i}}^{i}$ for $i=0,1, \ldots, n$, so it can remove the output masking bits to get $f\left(c_{0} \oplus r_{0}, \ldots, c_{n-1} \oplus r_{n-1}\right)=f\left(b_{0}, \ldots, b_{n-1}\right)$.

Unfortunately, while the protocol above turns out to be secure when only the evaluator is corrupted, it does not satisfy our notion of security. Consider a 3-argument function $f\left(x_{0}, x_{1}, x_{2}\right)$, where on input $f\left(0, x_{1}, x_{2}\right)$ the outputs are split to three 0 's and a single 1 , and for $f\left(1, x_{1}, x_{2}\right)$ the outputs are balanced 2-2. If the evaluator corrupts $P_{2}$ (the party of the last level of the tree) and receives its outputmasking bits, it immediately learns whether the right side of the tree corresponds to $f\left(0, x_{1}, x_{2}\right)$ or to $f\left(1, x_{1}, x_{2}\right)$. This is due to the fact that the output-masking bits up to that level are the same for the sub tree with the two 0's. Thus, the last output-masking bit reveals these values. When the adversary gets $c_{0}$ from $P_{0}$, it immediately knows whether its input is 0 or 1 .

\subsubsection{A Secure Solution}

It turns out that the insecure protocol from above can be made secure by using per-leaf output-masking bits rather than per-edge. Namely, each $P_{i}$ still gets one input-masking bit $r_{i}$, but now it gets $2^{n}$ output-masking bits, one for each leaf in the tree, $s_{c_{0}, \ldots, c_{n-1}}$ for all $\left(c_{0}, \ldots, c_{n-1}\right) \in\{0,1\}^{n}$. The table given to the evaluator also changes accordingly, namely for each leaf with name $c_{0}, \ldots, c_{n-1}$ the table holds the value $f\left(c_{0} \oplus r_{0}, \ldots, c_{n-1} \oplus r_{n-1}\right) \oplus s_{c_{0}, \ldots, c_{n-1}}^{0} \oplus s_{c_{0}, \ldots, c_{n-1}}^{1} \oplus$ $\cdots \oplus s_{c_{0}, \ldots, c_{n-1}}^{n-1}$.

The protocol is modified to reflect the above change as follows. On input $b_{i}$, party $P_{i}$ sets $c_{i} \leftarrow b_{i} \oplus r_{i}$, then it assembles a subset of its output-bit masks that are consistent with $c_{i}$; namely, $S_{i}=\left\{s_{e_{0}, \ldots, e_{i-1}, c_{i}, e_{i+1}, \ldots, e_{n-1}}^{i}\right\}$ for all $e_{0}, \ldots, e_{i-1}, e_{i+1}, \ldots, e_{n-1} \in\{0,1\}^{n-1}$ (in order), and sends $\left(c_{i}, S_{i}\right)$ to the evaluator. As before, the bits $c_{0}, \ldots, c_{n-1}$ that the evaluator $\mathcal{E}$ receives define a complete root-to-leaf path in the tree, and $\mathcal{E}$ has all the associated output-masking bits $s_{c_{1}, \ldots, c_{n}}^{i}$, so it can remove those masks to get $f\left(c_{0} \oplus\right.$ $\left.r_{0}, \ldots, c_{n-1} \oplus r_{n-1}\right)=f\left(b_{0}, \ldots, b_{n-1}\right)$. It is easy to see that the different masking for each leaf prevent the adversary from analyzing the tree, yielding the following lemma.

LEMmA 3.2. For any n-bit-input function $f$, the above star-compliant protocol for function $f$, is secure in the semihonest model. Each party gets at most $2^{n}+1$ bits of correlated randomness and sends at most $2^{n-1}+1$ bits.

Proof. Let $T=\left\{i_{1}, \ldots, i_{l}\right\}$ be the corrupted parties. The simulator has the corrupted-parties' input and the residual function. For the star pattern, all the corrupted parties are free, so the simulator gets all the values $f\left(b_{0}, \ldots, b_{n-1}\right)$ where the honest parties' input is fixed and the corrupted parties' input vary over all $2^{l}$ possibilities.

To compute the table for $\mathcal{E}$, the simulator first chooses random bits $r_{0}, \ldots, r_{n-1}$ and also random bits $s_{c_{0}, \ldots, c_{n-1}}^{i}$ for all $i \leq n$ and $c_{0}, \ldots, c_{n-1} \in\{0,1\}^{n}$. It chooses a vector of inputs for the honest parties which is consistent with the inputs of the faulty parties and the residual function; denote by $b_{i}$ the input chosen for the honest party $P_{i}$. For every value of the residual function related to $e_{i_{1}}, \ldots, e_{i_{l}}$, the simulator computes the leaf to which this value will be assigned as follows: for a fixed input, if the party is honest then it uses the value $b_{i}$ it chose for it and if the party is faulty it uses the value it got from the adversary, and sets $c_{i}=b_{i} \oplus r_{i}$. For the free inputs, it sets $c_{i}=e_{i} \oplus r_{i}$. It will compute the value for the table at location $c_{0}, \ldots, c_{n-1}$ by taking the value $d$, from the residual function associated with $e_{i_{1}}, \ldots, e_{i_{l}}$ and computing $d \oplus s_{c_{0}, \ldots, c_{n-1}}^{0} \oplus \ldots \oplus s_{c_{0}, \ldots, c_{n-1}}^{n-1}$.

Once all the values at the locations associated with the residual function have been entered, the simulator completes populating the table for $\mathcal{E}$ with random values.

It computes the view for the adversary by running the computation on the fixed inputs that it had chosen and all the random values. The result of this computation is given to the adversary. It includes all the randomness that relates to the faulty parties (whether their inputs are fixed or free), the table for $\mathcal{E}$ and all the values that the honest parties would transfer to $\mathcal{E}$ during the execution as computed above.

In the real execution for an honest party $P_{i}$ on input $b_{i}^{\prime}$, the simulator fixes $r_{i}^{\prime}$ to be $b_{i} \oplus r_{i} \oplus b_{i}^{\prime}$. Furthermore, it uses the paddings for the leaves that it chose above. This would result in an identical execution for any input vectors of the honest parties that are consistent with the values of the residual function.

\subsubsection{Extending the Star Protocol to General DAGs}

Although we can use the reduction from Section 2 to transform the star protocol to a protocol for any other patterns, below we describe a direct approach that yields more efficient protocols for DAG patterns.

The format of the decision tree and its randomness for the case of the DAG are identical to the star, except that we require the ordering of parties to be consistent with the topological order on the DAG. (Namely each party is assigned to a level of the tree larger than that of all its predecessors in the DAG, and smaller than that of all its successors.) Again, we will call the node assigned to level $i$ party $P_{i}$, and give it the input-masking bit $r_{i}$ and the leaf-masking randomness for each leaf in the tree. Also the evaluator $\mathcal{E}$ is given exactly the same table as in the star protocol above, namely 
for each leaf with name $c_{0}, \ldots, c_{n-1}$ the table holds the value $f\left(c_{0} \oplus r_{0}, \ldots, c_{n-1} \oplus r_{n-1}\right) \oplus s_{c_{0}, \ldots, c_{n-1}}^{0} \oplus \ldots \oplus s_{c_{0}, \ldots, c_{n-1}}^{n-1}$.

The Protocol. The difference between the star protocol and the general-DAG protocol is that $P_{i}$ that gets sets of output-masking bits $S^{i^{\prime}}$ from other parties upstream $\left(i^{\prime}<i\right)$ will prune them to be consistent with its input and with each other before forwarding these sets downstream toward the evaluator ${ }^{5}$ Party $P_{0}$ computes $c_{0}=b_{0} \oplus r_{0}$, a sequence of labeled output-masking bits

$S_{0}=\left\{\left\langle\left(c_{0}, e_{1}, \ldots, e_{m}\right): s_{c_{0}, e_{1}, \ldots, e_{m}}^{0}\right\rangle \mid\left(e_{1}, \ldots, e_{m}\right) \in\{0,1\}^{m}\right\}$

where $m=n-1$, and a pattern-vector $V_{0}=\left(c_{0}, *, \ldots, *\right)$ that reflects the coordinates that $P_{0}$ knows to be fixed. As it is at the root of the tree it only knows its own coordinate. $P_{0}$ sends $\left(0, V_{0}, S_{0}\right)$ to all the nodes that it can reach in the DAG.

Party $P_{i}$ receives a collection of tuples $\left(i_{j}, V_{i_{j}}, S_{i_{j}}\right)$ belonging to parties upstream from it, $i_{1}, \ldots, i_{l}<i$. (It may get multiple tuples $\left(i_{j}, \star, \star\right)$ for the same $i_{j}$ on several incoming edges, since this is a semi-honest execution then the corresponding $V_{i_{j}}, S_{i_{j}}$ can only differ by having different pruning.) Each vector $V_{i_{j}}$ reflects inputs that a node upstream knows to be fixed, and hence represents some pruning of previous values.

Party $P_{i}$ creates the "merged vector" $V_{i}$, in which a coordinate $k$ has $V_{i}[k]=*$ if and only if (a) $V_{i_{j}}[k]=*$ in all the incoming vectors $V_{i_{j}}$, and (b) $k \neq i$. It sets the $i$ 'th coordinate to be $V_{i}[i]=c_{i}$, and the value of every other non-star coordinate is taken from one of the incoming $V_{i_{j}}$ where this entry is not a $*$. (Again, since this is a semi-honest execution, then if $V_{i_{j}}[k] \neq *$ it must be the case that $V_{i_{j}}[k]=c_{k}$, i.e., this entry contains the $c_{k}$ value of the party who first set it to a non-* value.)

Then, $P_{i}$ prunes the sets of output-masking bits in all the $S_{i_{j}}$ 's to include only those consistent with $V_{i}$. In other words, denote the set of labels in $S_{i_{j}}$ by $X_{i_{j}}$, then the set of labels consistent with $V_{i}$ is the intersection of all the $X_{i_{j}}$ 's, further reduced to only the labels with the $i$ 'th bit equals $c_{i}$. Party $P_{i}$ then drops all the bits from each $S_{i_{j}}$ that are labeled by inconsistent labels. It also computes its own set of labeled output-masking bits

$S_{i}=\left\{\left\langle\left(e_{0}, \ldots, e_{m}\right): s_{e_{0}, \ldots, e_{m}}^{i}\right\rangle \mid\left(e_{0}, \ldots, e_{m}\right)\right.$ consistent with $\left.V_{i}\right\}$

where $m=n-1$ and sends on all its outgoing edges all the pruned $\left(V_{i}, S_{i_{j}}\right)$ 's together with its own $\left(V_{i}, S_{i}\right)$.

The evaluator applies exactly the same pruning procedure as before, and if all the nodes are upstream from it then this leaves a single labeled output-masking bit from every party $\left\langle\left(c_{0}, \ldots, c_{n-1}\right): s_{c_{0}, \ldots, c_{n-1}}^{i}\right\rangle$. The evaluator looks up the value that it has for the leaf $\left(c_{0}, \ldots, c_{n-1}\right)$ and unmask all the output-masking bits to get the function value $f\left(c_{0} \oplus\right.$ $\left.r_{0}, \ldots, c_{n-1} \oplus r_{n-1}\right)=f\left(b_{0}, \ldots, b_{n-1}\right)$. This completes the description of the protocol.

Lemma 3.3. For any n-bit-input function $f$ and a $D A G$ pattern $\mathcal{I}$, the above $\mathcal{I}$-compliant protocol for computing the function $f$ is semi-honest secure.

Proof. The proof for the DAG follows the exact same proof as for the case of the star with the addition that when

\footnotetext{
${ }^{5}$ This can be thought of as similar to what happens in the chain protocol, where only a single value needs to be forwarded, but the pruning in this protocol is not as efficient (even if the DAG happens to be a chain).
}

the simulator computes the execution of the protocol it also creates the vectors $V_{i}$ and does the same pruning. These vectors can be created in a straightforward manner from all the information which the simulator holds.

A simple change of the randomness will yield an identical execution in the real and simulated worlds.

See the full version for the malicious security case.

\section{Acknowledgments}

We thank Mor Weiss for her useful comments on an earlier version of this manuscript.

This work was done in part while the authors were visiting the Simons Institute for the Theory of Computing, supported by the Simons Foundation and by the DIMACS/Simons Collaboration in Cryptography through NSF grant \#CNS1523467. First and fifth authors supported in part by the Defense Advanced Research Projects Agency (DARPA) and Army Research Office (ARO) under Contract No. W911NF15-C-0236. Second author supported by ERC grant 259426, a DARPA/ARL SAFEWARE award, NSF Frontier Award 1413955, NSF grants 1228984, 1136174, 1118096, and 1065276, and by the DARPA through the ARL under Contract W911NF15-C-0205. Second and fourth authors supported by ISF grant 1709/14 and BSF grant 2012378. Third author supported in part by DARPA/ARO Safeware Grant \# W911NF15-C-0213. The views expressed are those of the authors and do not reflect the official policy or position of the Department of Defense, the National Science Foundation, or the U.S. Government.

\section{REFERENCES}

[1] S. Agrawal, S. Gorbunov, V. Vaikuntanathan, and H. Wee. Functional encryption: New perspectives and lower bounds. In CRYPTO, pages 500-518, 2013.

[2] B. Barak, O. Goldreich, R. Impagliazzo, S. Rudich, A. Sahai, S. P. Vadhan, and K. Yang. On the (im)possibility of obfuscating programs. J. ACM, $59(2): 6,2012$.

[3] A. Beimel, A. Gabizon, Y. Ishai, E. Kushilevitz, S. Meldgaard, and A. Paskin-Cherniavsky. Non-interactive secure multiparty computation. In CRYPTO, pages 387-404, 2014.

[4] M. Bellare, V. T. Hoang, and P. Rogaway. Foundations of garbled circuits. In $A C M C C S$, pages 784-796, 2012.

[5] M. Ben-Or, S. Goldwasser, and A. Wigderson. Completeness theorems for non-cryptographic fault-tolerant distributed computation. In $S T O C$, pages 1-10, 1988.

[6] R. Bendlin, I. Damgård, C. Orlandi, and S. Zakarias. Semi-homomorphic encryption and multiparty computation. In EUROCRYPT, pages 169-188, 2011.

[7] D. Boneh, A. Sahai, and B. Waters. Functional encryption: Definitions and challenges. In $T C C$, pages 253-273, 2011.

[8] D. Boneh and A. Silverberg. Applications of multilinear forms to cryptography. IACR Cryptology ePrint Archive, 2002:80, 2002.

[9] R. Canetti. Security and composition of multiparty cryptographic protocols. J. Cryptology, 13(1):143-202, 2000.

[10] A. D. Caro, V. Iovino, A. Jain, A. O'Neill, O. Paneth, and G. Persiano. On the achievability of 
simulation-based security for functional encryption. In CRYPTO, pages 519-535, 2013.

[11] N. Chandran, J. A. Garay, and R. Ostrovsky. Improved fault tolerance and secure computation on sparse networks. In ICALP, pages 249-260, 2010.

[12] D. Chaum, C. Crépeau, and I. Damgård. Multiparty unconditionally secure protocols. In STOC, pages 11-19, 1988.

[13] B. Chor, E. Kushilevitz, O. Goldreich, and M. Sudan. Private information retrieval. J. ACM, 45(6):965-981, 1998.

[14] J. Coron, T. Lepoint, and M. Tibouchi. Practical multilinear maps over the integers. In CRYPTO, pages 476-493, 2013.

[15] J. Coron, T. Lepoint, and M. Tibouchi. New multilinear maps over the integers. In CRYPTO, pages $267-286,2015$.

[16] I. Damgård, V. Pastro, N. P. Smart, and S. Zakarias. Multiparty computation from somewhat homomorphic encryption. In CRYPTO, pages 643-662, 2012.

[17] I. Damgård and S. Zakarias. Constant-overhead secure computation of boolean circuits using preprocessing. In TCC, pages 621-641, 2013.

[18] Y. Dodis, J. Katz, L. Reyzin, and A. Smith. Robust fuzzy extractors and authenticated key agreement from close secrets. In CRYPTO, pages 232-250, 2006.

[19] D. Dolev, C. Dwork, O. Waarts, and M. Yung. Perfectly secure message transmission. J. ACM, 40(1):17-47, 1993.

[20] C. Dwork, D. Peleg, N. Pippenger, and E. Upfal. Fault tolerance in networks of bounded degree. SIAM J. Comput., 17(5):975-988, 1988.

[21] U. Feige, J. Killian, and M. Naor. A minimal model for secure computation. In STOC, pages 554-563, 1994.

[22] J. A. Garay and R. Ostrovsky. Almost-everywhere secure computation. In EUROCRYPT, pages 307-323, 2008.

[23] S. Garg, C. Gentry, and S. Halevi. Candidate multilinear maps from ideal lattices. In EUROCRYPT, pages 1-17, 2013.

[24] S. Garg, C. Gentry, S. Halevi, M. Raykova, A. Sahai, and B. Waters. Candidate indistinguishability obfuscation and functional encryption for all circuits. In FOCS, pages 40-49, 2013.

[25] C. Gentry. Fully homomorphic encryption using ideal lattices. In STOC, pages 169-178, 2009.

[26] C. Gentry, S. Gorbunov, and S. Halevi. Graph-induced multilinear maps from lattices. In $T C C$, pages 498-527, 2015.

[27] O. Goldreich. The Foundations of Cryptography Volume 2, Basic Applications. Cambridge University Press, 2004.
[28] O. Goldreich, S. Micali, and A. Wigderson. How to play any mental game or a completeness theorem for protocols with honest majority. In $S T O C$, pages 218-229, 1987.

[29] S. Goldwasser, S. D. Gordon, V. Goyal, A. Jain, J. Katz, F. Liu, A. Sahai, E. Shi, and H. Zhou. Multi-input functional encryption. In EUROCRYPT, pages 578-602, 2014.

[30] S. Goldwasser, Y. T. Kalai, R. A. Popa, V. Vaikuntanathan, and N. Zeldovich. Reusable garbled circuits and succinct functional encryption. In STOC, pages 555-564, 2013.

[31] S. Goldwasser, S. Micali, and C. Rackoff. The knowledge complexity of interactive proof systems. SIAM J. Comput., 18(1):186-208, 1989.

[32] S. D. Gordon, T. Malkin, M. Rosulek, and H. Wee. Multi-party computation of polynomials and branching programs without simultaneous interaction. In EUROCRYPT, pages 575-591, 2013.

[33] S. Halevi, Y. Lindell, and B. Pinkas. Secure computation on the web: Computing without simultaneous interaction. In CRYPTO, pages 132-150, 2011.

[34] P. Hubacek and D. Wichs. On the communication complexity of secure function evaluation with long output. In ITCS, pages 163-172, 2015.

[35] Y. Ishai, M. Prabhakaran, and A. Sahai. Founding cryptography on oblivious transfer - efficiently. In CRYPTO, pages 572-591, 2008.

[36] M. Kearns, J. Tan, and J. Wortman. Network-faithful secure computation.

[37] J. Kilian. Founding cryptography on oblivious transfer. In STOC, pages 20-31, 1988.

[38] M. Naor and K. Nissim. Communication preserving protocols for secure function evaluation. In $S T O C$, pages 590-599, 2001.

[39] A. O'Neill. Definitional issues in functional encryption. IACR Cryptology ePrint Archive, 2010:556, 2010.

[40] A. Sahai and B. Waters. Fuzzy identity-based encryption. In EUROCRYPT, pages 457-473, 2005.

[41] N. B. Shah, K. V. Rashmi, and K. Ramchandran. Secret share dissemination across a network. CoRR, abs/1207.0120, 2012.

[42] A. Srinivasan and D. Zuckerman. Computing with very weak random sources. SIAM J. Comput., 28(4):1433-1459, 1999.

[43] A. Yao. Protocols for secure computations. In FOCS, pages 160-164, 1982.

[44] A. C.-C. Yao. How to generate and exchange secrets. In FOCS, pages 162-167, 1986. 